\title{
Synthesis of a Telithromycin Analogue
}

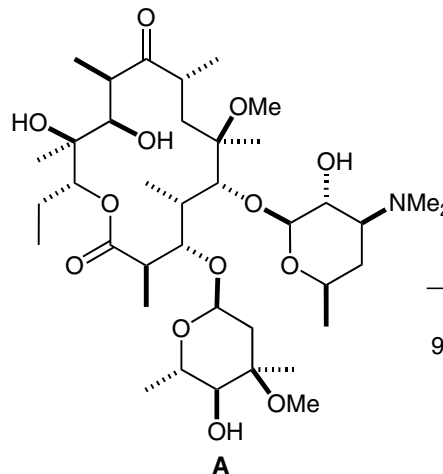

Clarithromycin
2 steps $98 \%$ (67 mol scale)<smiles>CC(=O)n1ccnc1</smiles>

. $\mathrm{HCl}$ to $\mathrm{pH} 2, \mathrm{EtOH}-\mathrm{H}_{2} \mathrm{O}, 38^{\circ} \mathrm{C}, 18 \mathrm{~h}$

2. $\mathrm{SMe}_{2}$ (1.05 equiv), NCS (1.0 equiv) $\mathrm{Et}_{3} \mathrm{~N}$ (0.55 equiv), $\mathrm{CH}_{2} \mathrm{Cl}_{2},-10^{\circ} \mathrm{C}$ (34 mol scale)

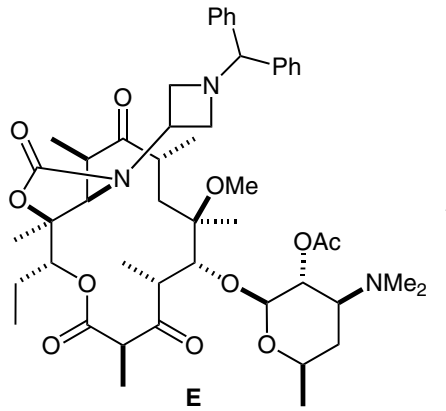

1. $\mathrm{MeOH}, \Delta, 12 \mathrm{~h}$

2. $\mathrm{H}_{2}(3.46 \mathrm{bar}), 20 \% \mathrm{Pd}(\mathrm{OH})_{2}$ $\mathrm{HCl}$ to $\mathrm{pH} 3.8-4.2$ $\mathrm{MeOH}, 50^{\circ} \mathrm{C}, 2 \mathrm{~h}$
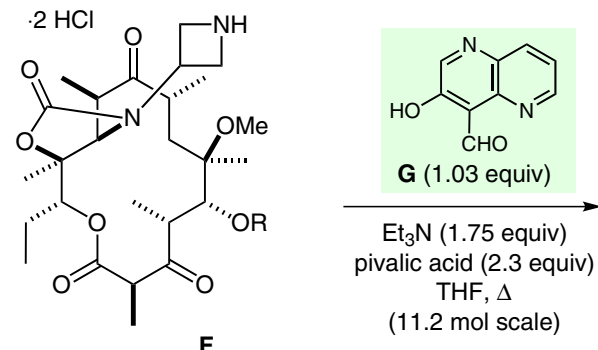

1. $\mathrm{NaBH}(\mathrm{OAc})_{3}$ (2.5 equiv) $\mathrm{MeCN}-\mathrm{EtOAc}-\mathrm{THF}$, r.t.

2. reverse phase column chromatography 3. $\mathrm{TsOH} \cdot \mathrm{H}_{2} \mathrm{O}$ (1.1 equiv) $44 \%$ from $\mathrm{F}$

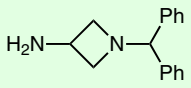

C (1.05 equiv)

DBU (3.0 equiv) $\mathrm{MeCN}, 50^{\circ} \mathrm{C}, 18 \mathrm{~h}$ (53 mol scale)
Synthesis of Natural

Products and

Potential Drugs

\section{Key words}

telithromycin analogues

clarithromycin

ketolide antibiotics

Corey-Kim

oxidation

reductive amination

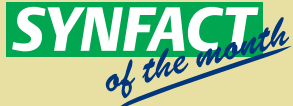

Significance: The target telithromycin analogue was under development as an oral antibiotic for the treatment of respiratory infections. The route depicted delivered kilogram quantities of API starting with clarithromycin (A).

sYNFACTS Contributors: Philip Kocienski

Synfacts 2012, 8(8), 0815 Published online: 19.07.2012

Dol: 10.1055/s-0032-1316597; Reg-No.: K04412SF
Comment: Note the use of a large-scale CoreyKim oxidation for the synthesis of the ketolide $\mathbf{E}$. An efficient large-scale synthesis of 3-hydroxy1,5-naphthyridine-4-carbaldehyde (G) was developed using a Skraup reaction. 